\title{
Population studies of diet and obesity
}

\author{
Lauren Lissner ${ }^{1 *}$, Berit L. Heitmann ${ }^{2}$ and Calle Bengtsson ${ }^{3}$ \\ ${ }^{1}$ Department of Internal Medicine, Göteborg University, Vasa Hospital, SE-41133 Gothenburg, Sweden \\ ${ }^{2}$ Unit for Dietary Studies, Copenhagen County Centre for Preventive Medicine, Glostrup University Hospital, Denmark \\ ${ }^{3}$ Department of Primary Health Care, Göteborg University, Gothenburg, Sweden
}

\begin{abstract}
Population-based research on diet, obesity and the metabolic syndrome is faced with accumulating evidence of biases that may profoundly affect results. One potential source of bias, which is often neglected in nutritional epidemiology, arises from self-selected study populations. Subjects who agree to participate in surveys may be at less risk of metabolic syndrome than those who refuse. Analogous to observations in adult populations, studies of schoolchildren have also yielded clear evidence of self-selection. Whether such selection patterns influence analytical results depends on how the biases relate to the dependent and independent variables being studied. Systematic dietary reporting error is another source of bias in studies of nutritional risk factors for disease. While obesity-related under-reporting bias is now well documented, less is known about whether specific foods and nutrients are disproportionately affected. However, two studies employing biomarkers for protein have suggested that obese subjects under-reported the proportion of energy from fat plus carbohydrate. This should alert epidemiologists to the possibility that a dual reporting bias may be present in studies of diet and disease: general under-reporting among obese subjects compounded by food-specific errors. In summary, biases due to self-selection and selective dietary under-reporting may produce consequences in epidemiological studies that are both unpredictable and complex. We conclude this review with recent findings involving dietary fat intake and regional adiposity in a population-based study of women. These preliminary results may have etiological relevance to the development of metabolic syndrome, but multiple biases of the type described previously may also be operating.
\end{abstract}

Obesity: Metabolic syndrome: Under-reporting: Self-selection: Bias

\section{Introduction}

Knowledge of dietary determinants of obesity is fundamental to our management of the current epidemic, yet there is accumulating evidence that profound biases exist in population studies of diet and obesity. The most widely acknowledged of these involves dietary reporting error, a problem that will be a main focus of this paper. Also of importance is the presence of bias due to self-selection among participants in population studies. Finally, we present a new analysis involving dietary fat intake in a population-based study, which may be relevant to development of the metabolic syndrome.

\section{Self-selected participants}

Selection bias is a well known problem in population-based research. Subjects who agree to participate in surveys are likely to be different from those who choose not to, and it is not understood how self-selection affects the diet-obesity association. Firstly, it is interesting to consider examples of obesity-related selection bias in population-based research. For instance, the Prospective Population Study of Women in Gothenburg, Sweden had a high participation rate at the baseline exam $(>90 \%)$ but has since experienced some attrition, even among former participants still living in Gothenburg. Examination of baseline anthropometry in relation to drop-out in the cohort 12 years later revealed a trend towards a higher waist-hip ratio among women who subsequently became non-participants (Bengtsson et al. 1989). Specifically, the mean waist-hip ratio of participants was 0.74 while that of non-participants was $0.75(P<0.05)$. No significant differences were observed in serum triglyceride or cholesterol concentrations. However, marked contrasts in systolic blood pressure were observed (132 versus $138 \mathrm{~mm} \mathrm{Hg}$ in participants versus non-participants, $P<$ 0.001). These results are summarized in Table 1. Similarly, data from the Danish draft board examination involving the complete cohort of young males showed that obesity in young adulthood, as assessed by the draft board, is a strong predictor for subsequent non-participation in health

\footnotetext{
*Corresponding author: L. Lissner, fax +46 31 7781704, email lauren.lissner@medfak.gu.se
} 
examinations. Almost $50 \%$ of those who were obese at the draft board examination, as opposed to only $20 \%$ of normalweight subjects, were non-participants in a health examination performed 4-40 years later (Sonne-Holm et al. 1989). Thus epidemiological surveys involving obesity, central obesity and other features of the metabolic syndrome may be missing an important portion of the population distribution.

Turning to diet, a more recent study was able to examine consequences of non-participation in a survey of dietary habits and attitudes in Swedish adolescents (Berg et al. 1998). Because the first stage of the dietary survey was conducted at school in a supervised manner, initial participation was virtually complete. However, in the secondary portion of the survey only $69 \%$ of schoolchildren completed the protocol. Characteristics of these non-participating subjects (e.g. older, smokers, single-parent families, etc.) are given in Table 1 . In addition to characterizing types of schoolchildren who are most likely to be non-participants, the subsequent drop-outs in the secondary dietary recordkeeping part of the survey made it possible to describe the biased results that would be obtained from using the reduced (secondary) sample. Notably, gender differences in skipping breakfast were exaggerated as a consequence of self-selection, whereas gender differences in fat content of milk products were obscured.

Lower smoking rates in participants compared to refusers, which we have also observed in an ongoing Swedish adult population (unpublished observations), may complicate the interpretation of studies involving obesity, due to associations between smoking, leanness, and central fat distribution. From the studies named above and numerous others, it may be suggested that biased participation is most likely to affect descriptive epidemiology, i.e. prevalences and means, but can also distort analytical results. The latter might occur when the selection is biased in ways that relate to both the exposure and the outcome of interest. Although non-participation is impossible to avoid in population studies, careful attention must be paid to characteristics of drop-outs whenever feasible.

\section{Dietary reporting errors and obesity}

An extremely problematic source of bias in studies of diet and obesity is under-reporting. Nutritional epidemiologists generally agree on several principles concerning dietary reporting errors. Firstly, energy intake is on average underestimated, and recall-based methods seem particularly susceptible to under-reporting due to dependence on memory. Secondly, 'social desirability' may further influence both what subjects actually eat and what they report eating (Hebert et al. 1995). Finally, obesity represents a welldocumented example of under-reporting bias which may derive from (i) changes in true intake as a function of recording or being observed; (ii) lack of awareness concerning food items and amounts consumed; and/or (iii) reluctance to disclose foods or amounts eaten.

It is not well documented whether some food types are selectively under-reported in the general population; here we consider the situation in obese subjects, among whom under-reporting has been most clearly demonstrated. In a population of Danish men and women who reported their usual diets while collecting urine samples for nitrogen assessment, obesity-related under-reporting was confirmed in relation to energy requirement estimated from body composition and physical activity. Additionally, there was proportionally less under-reporting of protein compared to other energy sources combined, providing evidence that foods high in fat and/or carbohydrates were being underreported more (Heitmann \& Lissner 1995). A similar trend has been observed in Swedish subjects (Lissner et al. 1998), although these data are less convincing of a macronutrientrelated bias given that obesity-related under-reporting is not observed with this particular method. However, these two

Table 1. Characteristics of non-participants in two Swedish studies*

\begin{tabular}{|c|c|c|}
\hline Study & $\begin{array}{l}\text { Non-participants } \\
\text { differed from } \\
\text { participants with } \\
\text { respect to: }\end{array}$ & $\begin{array}{l}\text { Non-participants } \\
\text { were similar to } \\
\text { participants with } \\
\text { respect to: }\end{array}$ \\
\hline $\begin{array}{l}\text { Population study of women from } \\
\text { Gothenburg, Sweden (Bengtsson } \\
\text { et al. 1989) }\end{array}$ & $\begin{array}{l}\text { Waist-hip ratio (higher) } \\
\text { Systolic blood pressure } \\
\text { (higher) }\end{array}$ & $\begin{array}{l}\text { Height } \\
\text { Weight } \\
\text { Cholesterol } \\
\text { Triglycerides } \\
\text { Hemoglobin }\end{array}$ \\
\hline $\begin{array}{l}\text { Dietary survey of fifth, seventh and } \\
\text { ninth graders in Mölndal, Sweden } \\
\text { (Berg et al. 1998) }\end{array}$ & $\begin{array}{l}\text { Gender (male) } \\
\text { Age (older) } \\
\text { Family (non-nuclear) } \\
\text { Parental origin } \\
\text { (immigrants) } \\
\text { Tobacco (user) } \\
\text { TV and video watching } \\
\text { (more) } \\
\text { Breakfast (skips it) } \\
\text { Whole milk (intention to drink) }\end{array}$ & $\begin{array}{l}\text { Exercise } \\
\text { Video game usage } \\
\text { Birth in Sweden } \\
\text { Milk at breakfast } \\
\text { Intention to eat } \\
\text { whole-grain bread } \\
\text { Intention to drink } \\
\text { low-fat milk } \\
\text { Intention to drink } \\
\text { medium-fat milk }\end{array}$ \\
\hline
\end{tabular}

\footnotetext{
* Both are two-stage surveys in which participation during the initial phase was almost complete. In the firs study, 38-60-year-old participants at baseline examination were categorized with respect to participation in the 12-year follow-up survey. In the second study, respondents to an in-school questionnaire were subsequently asked to complete a detailed food record.
} 
Table 2. Characteristics of participants in dietary surveys (mean + SD unless noted otherwise)

\begin{tabular}{lccc}
\hline & \multicolumn{3}{c}{ Period examined $(N=271)$} \\
\cline { 2 - 4 } Characteristics & \multicolumn{1}{c}{$1968 / 69$} & $1974 / 75$ & $1980 / 81$ \\
\hline Age $(\mathrm{range})$ & $38-60$ & $44-66$ & $50-72$ \\
$\mathrm{BMI}\left(\mathrm{kg} / \mathrm{m}^{2}\right)$ & $24.4(3.9)$ & $25 \cdot 1(4.0)$ & $25.5(4.0)$ \\
Waist-hip ratio (cm/cm) & $0.74(0.05)^{*}$ & $0.79(0.07)$ & $0.81(0.07)$ \\
Cigarettes per day & $4.2(6.6)$ & $4.3(6.7)$ & $3.4(6.4)$ \\
Percentage sedentary at leisure & 13.7 & 17.7 & 28.0 \\
Percentage consuming alcohol weekly & 53.5 & 49.1 & 34.7 \\
Energy-per cent derived from fat & $38.0(5.5)$ & $35.9(4.9)$ & Not included \\
\hline
\end{tabular}

* Missing data for one subject.

consistent observations should alert epidemiologists to the possibility that a dual bias may be present in studies of diet and disease: general under-reporting among obese subjects, compounded by food-specific under-reporting. Interestingly, recent data from Denmark suggest that the nature of this bias may be changing over time, such that social desirability may affect reported nutrient intakes in the whole population, not only in obese groups (Heitmann et al. 2000).

How can the problem of selective under-reporting be more accurately documented? The further use of biomarkers is necessary as two subjective methods have dependent errors and thus yield uncertain comparative results. For instance, the combined use of urinary nitrogen as a marker for dietary protein and doubly labelled water as an indicator of energy intake could advance our understanding of non-protein reporting errors. Types of fat under-reporting can also be explored with the help of biomarkers such as fatty acid determinations from adipose tissue and blood samples.

Another approach to studying selective under-reporting involves comparing reported diets with observations of true intake. It has been reported in several countries that per capita fat intake from food consumption surveys is lower than estimates from national food disappearance data. Conversely, Radimer \& Harvey (1998) reported that an Australian community undergoing a cardiovascular disease intervention over-reported their usual consumption of fatand salt-reduced foods, compared to local sales figures. Other studies have conducted covert observation of subjects in a quasi-natural laboratory situation. For instance, researchers in Cambridge covertly recorded the true intakes of 33 women who were being observed in a metabolic facility (Poppit et al. 1998). After the study was completed, $24 \mathrm{~h}$ recalls were conducted during which subjects most frequently failed to record their between-meal snacks. Only $64 \%$ of the energy from snacks was reported, in comparison to $96 \%$ of energy from meals. As expected, total energy intake was under-reported, but the only significantly underreported macronutrient was carbohydrate ( $80 \%$ of observed). Finally, in contrast to most previous studies, the magnitude of under-reporting was not higher in the obese women. Using a similar approach at the Laboratory of Clinical Metabolism in Gothenburg, we also observed no obesityrelated under-reporting in men, although certain foodspecific errors of omission were detected across weight groups, e.g. failing to report butter spread and foods in the fruit/vegetable category (unpublished observations). Thus the classic obesity-related reporting bias may be modified by subjects' attention to what they are being fed, together with awareness that they are being observed.

Systematic or non-random errors of the type investigated in the above-mentioned studies could have important implications in nutritional epidemiology. For instance, if obese subjects truly overconsume high-fat foods but selectively under-report them, a simple consequence might be that the overconsumption is not detected. However, other consequences are less clear, for instance when obesity is both a source of bias in the dietary exposure and a confounding factor for a disease being studied. Here, selective underreporting of fat intake by obese individuals may produce consequences in analytical epidemiological studies that are both unpredictable and complex.

\section{Example: dietary fat and waist-hip ratio in a population studied longitudinally}

In view of the situation described above, it is legitimate to pose the question whether population-based research involving diet and obesity can be pursued at all. We propose that the answer is yes, with the caveat that analyses be considered exploratory. We present an example of diet in relation to the waist-hip ratio as observed in a subsample (Table 2) of the Population Study of Women in Gothenburg, a prospective study of randomly selected Swedish women initially aged 38-60 years (Bengtsson et al. 1989).

In two studies, no associations were observed between dietary fat and obesity in the whole cohort, except in certain susceptible subgroups of sedentary women (Lissner et al. 1997) and those with a positive parental history of obesity (Heitmann et al. 1995). Given the longitudinal design of the study we have taken the opportunity to examine associations between changes in dietary fat during the initial 6-year observation period, in relation to changes in anthropometric measures in the subsequent 6-year period, in a random subsample of 271 women completing the dietary history interview at two points in time. Specifically, we focus on changes in waist-hip ratio as an anthropometric outcome measure that is linked to the metabolic syndrome.

The results shown in Fig. 1 indicate that increases in waist-hip ratio vary as a direct function of previous changes in fat intake $(P<0.03)$. Women in all dietary groups tended to increase their waist-hip ratio during the second 6-year observation period, particularly those in quintiles 4 and 5 of dietary change who, on average, had increased their habitual energy-per cent fat by $4 \cdot 1$ and $6 \cdot 8 \%$, respectively, during 


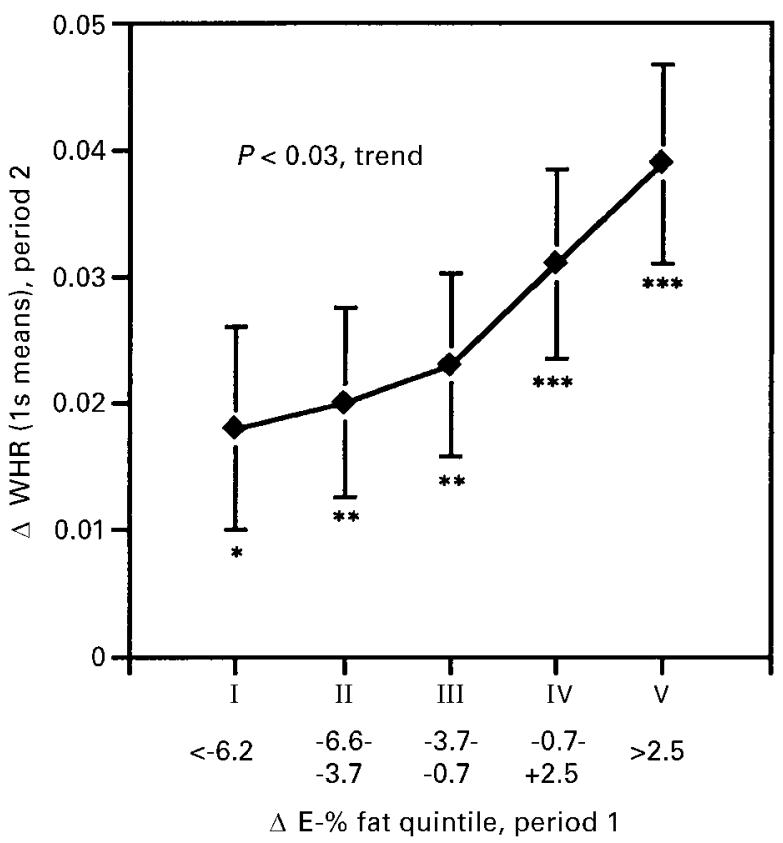

Fig. 1. Increases in waist-hip ratio (least-squares means) as a function of previous changes in energy-per cent fat among women initially aged $38-60$ and followed for 12 years. The $x$-axis represents quintiles/ranges of dietary change from 1968/69 to 1974/75; the $y$ axis shows mean increases in waist-hip ratio from 1974/75 to $1980 /$ 81 after adjustment for confounding factors. The continuous trend is significant at $P<0.03$. Asterisks refer to the significance of increases in waist-hip ratio over time in each dietary quintile group $\left({ }^{*} P<0.05\right.$; $\left.{ }^{\star *} P<0.01 ;{ }^{* \star *} P<0.0001\right)$.

the previous 6 years. This association has been controlled for potential confounders at baseline (age, waist-hip ratio, BMI, percentage dietary fat, smoking, alcohol, physical activity in 1968/69), at exam 2 (waist-hip ratio, BMI, smoking, alcohol, physical activity in 1974/ 75), and exam 3 (BMI, smoking, alcohol, physical activity in 1980/81).

To summarize, these data suggest that increases or decreases in usual fat intake may predict long-term development of central adiposity, which in turn is the strongest anthropometric risk factor for cardiovascular disease in this cohort (Bengtsson et al. 1993). The doubly prospective design used here strengthens arguments for causality by ensuring that the dietary changes are not consequences of the anthropometric changes. The extensive multivariate analysis adjusts for other obesityand lifestyle-related covariates that may be driving the association. While the dietary data are in all likelihood biased with respect to obesity (Hultén et al. 1990), this analysis assumes that the bias is relatively static within individuals, permitting them to be ranked with respect to dietary changes. In conclusion, this result may reflect a causal role for total fat intake in the development of central adiposity, or alternatively may be an example of a chance finding resulting from multiple underlying biases. It would be encouraging if these observations were to be replicated in other longitudinal population studies of diet and obesity.

\section{Acknowledgements}

The Prospective Population Study of Women in Gothenburg is funded in part by the Swedish Medical Research Council 27X-11653-02B. Christina Berg, Helene Berteus Forslund, and Lena Gripeteg are gratefully acknowledged for their contributions to the work reported here.

\section{References}

Bengtsson C, Björkelund C, Lapidus L \& Lissner L (1993) Associations of serum lipid concentrations and obesity with mortality in women -20 year follow up of participants in the prospective population study in Gothenburg, Sweden. British Medical Journal 307, 1385-1388.

Bengtsson C, Gredmark T, Hallberg L, Hällström T, Isaksson B, Lapidus L, Lindquist O, Lindstedt S, Lurie M, Nyström E, Rybo G, Samuelsson S, Ranfsson V \& Sigurdsson J (1989) The population study of women in Gothenburg 1980-81 - the third phase of a longitudinal study. Scandinavian Journal of Social Medicine 17, 141-145.

Berg C, Jonsson I, Conner MT \& Lissner L (1998) Sources of bias in a dietary survey of children. European Journal of Clinical Nutrition 52, 663-667.

Hebert J, Clemow L, Pbert L, Ockene IS \& Ockene JK (1995) Social desirability bias in dietary self-report may compromise the validity of dietary intake measures. International Journal of Epidemiology 24, 389-398.

Heitmann BL \& Lissner L (1995) Dietary under-reporting by obese individuals. Is it specific or nonspecific? British Medical Journal 311, 986-989.

Heitmann BL, Lissner L, Sørensen TIA \& Bengtsson C (1995) Dietary fat intake and weight gain in women genetically predisposed for obesity. American Journal of Clinical Nutrition $\mathbf{6 1}$, 1213-1217.

Heitmann BL, Lissner L \& Osler M (2000) Do we eat less fat, or just report so? International Journal of Obesity 24 (in press).

Hultén B, Bengtsson C \& Isaksson B (1990) Some errors in a longitudinal dietary survey revealed by the urine nitrogen test. European Journal of Clinical Nutrition 44, 169-174.

Lissner L, Heitmann BL \& Bengtsson C (1997) Low-fat diets may prevent weight gain in sedentary women: prospective observations from the Population Study of Women in Göteborg. Obesity Research 5, 43-48.

Lissner L, Heitmann BL \& Lindroos AK (1998) Measuring intake in free-living humans: a question of bias. Proceedings of the Nutrition Society 57, 333-339.

Poppitt SD, Swann D, Black AE \& Prentice AM (1998) Assessment of selective under-reporting of food intake by both obese and non-obese women in a metabolic facility. International Journal of Obesity 22, 303-311.

Radimer KL \& Harvey PWJ (1998) Comparison of self-report of reduced fat and salt foods with sales and supply data. European Journal of Clinical Nutrition 52, 380-382.

Sonne-Holm S, Sørensen TIA, Jensen G \& Schnohr P (1989) Influence of fatness, intelligence, education and sociodemographic factors on response rate in a health survey. Journal of Epidemiology and Community Health 43, 369-374. 